\title{
Dynamic Sampling of Cabin VOCs during the Mission Operations Test of the Deep Space Habitat
}

\author{
Osacar Monje \\ ESC Team QNA, Kennedy Space Center, FL, 32931 \\ and \\ Kristina Rojdev ${ }^{2}$ \\ NASA. Jet Propulsion Lab, CA, 91011
}

\begin{abstract}
The atmospheric composition inside spacecraft is dynamic due to changes in crew metabolism and payload operations. A portable FTIR gas analyzer was used to monitor the atmospheric composition of four modules (Core lab, Veggie Plant Atriun, Hygiene module, and Xhab loft) within the Deep Space Habitat (DSH) during the Mission Operations Test (MOT) conducted at the Johnson Space Center. The FTIR was either physically relocated to a new location or the plumbing was changed so that a different location was monitored. An application composed of $\mathbf{2 0}$ gases was used and the FTIR was zeroed using N2 gas every time it was relocated. The procedures developed for operating the FTIR were successful as all data was collected and the FTIR worked during the entire MOT mission. Not all the 20 gases in the application sampled were detected and it was possible to measure dynamic VOC concentrations in each DSH location.
\end{abstract}

\section{Nomenclature}

DSH = Deep Space Habitat

FTIR = Fourier Transform Infrared spectroscopy

ISS $=$ International Space Station

MOT $=$ Mission Operations Test

\section{Introduction}

$\mathrm{T}$ He knowledge gained from low-Earth orbit projects, such as the International Space Station (ISS), and Earthbased analog research from the Deep Space Habitat (DSH) will be used to expand human presence to remote environments, like an asteroid, Lagrange points, the moon or Mars. The Deep Space Habitat is a prototype of a future habitat that houses astronauts performing specific manned mission scenarios. The goal of the Deep Space Habitat is to evaluate habitation-related exploration technologies that will enable human exploration to multiple destinations. During the Mission Operations Test (MOT), four crew members lived inside the DSH at JSC for two weeks. The MOT simulated the return trip to Earth from an asteroid.

Trace contaminant control and monitoring technologies represent a significant developmental area for deep space exploration missions. Reliable, near real-time trace contaminant monitors and event monitors are needed for ensuring that the crew is not exposed to harmful chemicals. A portable FTIR spectrometer was deployed during the DSH MOT to continuosly record the composition of the air within the DSH. The data collected during the MOT will be used to develop future air revitalization subsystems, as the FTIR was used to identify and quantify the trace contaminant loads observed on DSH.

FTIR spectrometers record the interaction of IR radiation with a sample, measuring the frequencies at which the sample absorbs the radiation and the intensities of the absorption. Determining these frequencies allows identification of the sample's chemical make-up, since chemical functional groups are known to absorb radiation at

\footnotetext{
${ }^{1}$ Insert Job Title, Department Name, Address/Mail Stop, and AIAA Member Grade for first author.

${ }^{2}$ Insert Job Title, Department Name, Address/Mail Stop, and AIAA Member Grade for fourth author (etc).
} 
specific frequencies. The intensity of the absorption is related to the concentration of the component. Intensity and frequency of sample absorption are depicted in a two-dimensional plot called a spectrum.

An FTIR spectrometer obtains infrared spectra by first collecting an 'interferogram' of a sample signal with a Michelson type interferometer, which measures all infrared frequencies simultaneously to produce a spectrum.

A parallel, polychromatic radiation from an IR source is directed to an interferometer. In the interferometer the light passes through a beam splitter, which sends the light in two directions at right angles. One beam goes to a stationary mirror then back to the beam splitter. The other goes to a moving mirror. The motion of the mirror makes the total path length variable versus that taken by the stationary-mirror beam. When the two meet up again at the beam splitter, they recombine, but the difference in path lengths creates constructive and destructive interference pattern called an interferogram. The recombined beam passes through the sample. The sample absorbs all the different wavelengths characteristic of its spectrum, and this subtracts specific wavelengths from the interferogram. The detector now reports variation in energy versus time for all wavelengths simultaneously. A laser beam is superimposed to provide a reference for the instrument operation. The detected signal is digitized and Fourier transformed by the computer resulting in an IR spectrum of the sample gas. Although FTIR is able to analyse a large number of gases, the technique is not suitable for inert gases, homonuclear diatomic gases (e.g., $\mathrm{N}_{2}, \mathrm{Cl}_{2}, \mathrm{H}_{2}, \mathrm{~F}_{2}$ ).

FTIR has long been established as an accurate technology for the simultaneous measurement of gaseous emissions from industrial processes, thus, we wanted to investigate the feasibility of deploying a portable version for monitoring dyamic changes in composition occurring during a 2-week long manned mission. The Gasmet DX4040 analyser enables identification and quantification of multiple gaseous compounds simultaneously and accurately, with results available in seconds.

$\circ$ Why is FTIR in a deep space habitat important?

The portable FTIR gas analyser substantially reduces the amount of equipment required to monitor gases in the $\mathrm{DSH}$, but more importantly, the technology enables the simultaneous analysis of a large number of target compounds. The ability to measure dynamic changes in VOC concentrations in near-real time helps identify and quantify the VOCs present in manned habitats and reduces risk to the crew.

Previous work in testing air quality instruments on ISS has included the Volatile Organics Analyzer (VOA) from NASA and the Analysing Interferometer for Ambient Air (ANITA) from the European Space Agency [1-3]. VOA measurements are taken up to a few times a day, and so provide a snapshot of the presence (or absence) of some forty chemical species, but does not give insight into air constituent changes lasting less than several hours. ANITA measurements were taken more frequently, and the instrument was designed to run continuously.

\section{Materials and Methods}

\section{A. DSH dimensions}

The Deep Space Habitat is a one story, 4port habitat unit with an approximate volume of 56 cubic meters. The HDUDSH shell includes inflatable loft for additional habitation volume. The DSH was contained within Building 220 at JSC.

The shape of the DSH shell is cylindrical with a vertically oriented axis. It has a 5.0 meter inner diameter, 2.0 meter barrel height, and 0.65 meter end domes on top and bottom for total height of $3.3 \mathrm{~m}$. The DSH prototype shell is made of composite fiberglass resin-infused shell attached to 8 double $0.8 \mathrm{~cm}$ thick steel ribs.

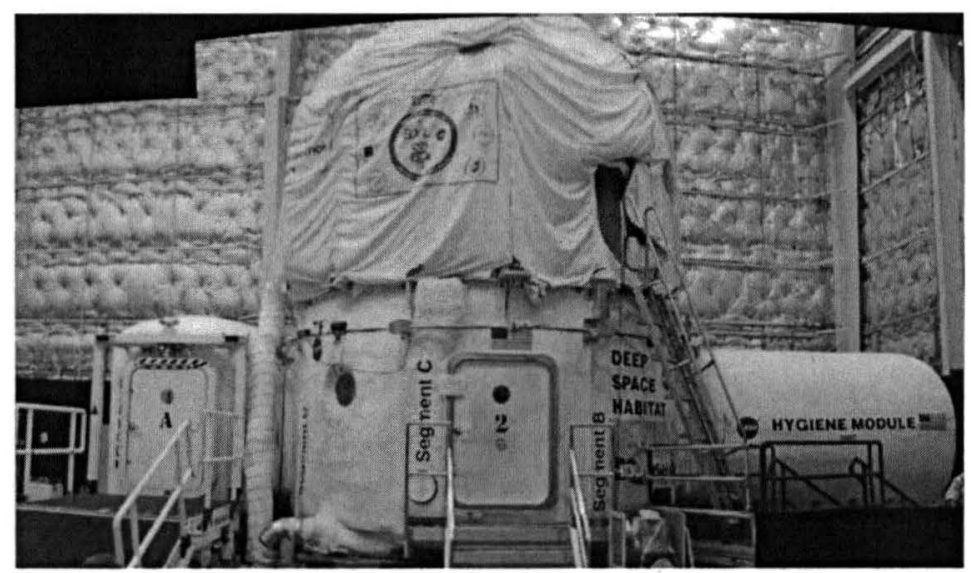




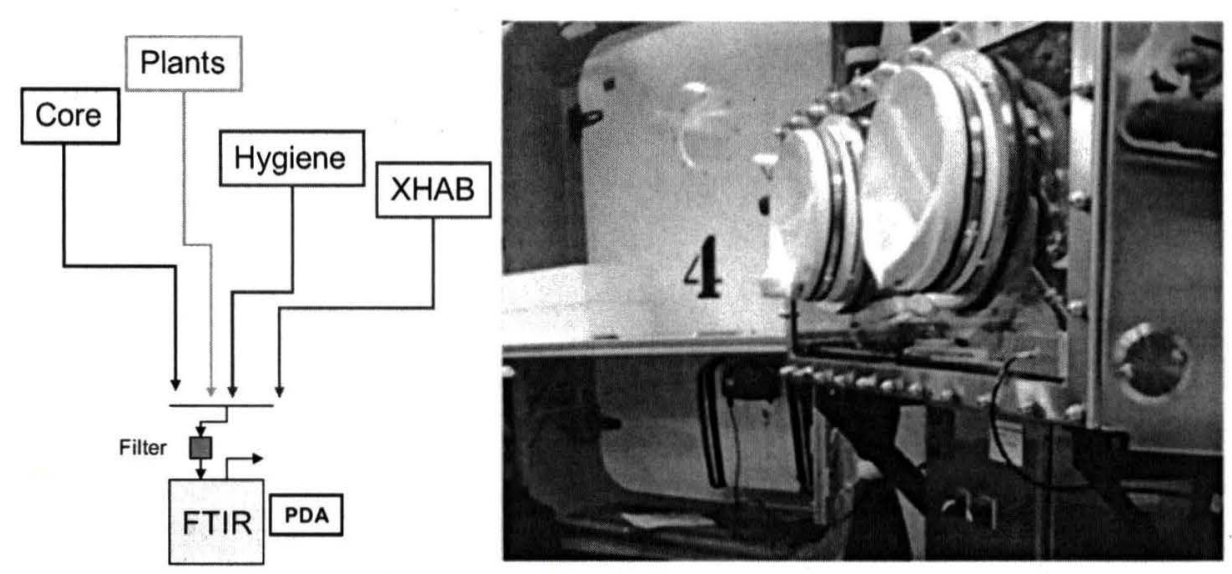

The FTIR spectrometer used was the Gasmet DX-4040, commercially available, portable gas analyzer. Procedures for zeroing the FTIR, for data collection and storage, and for moving the FTIR from location to location were established. The DSH MOT personnel were trained in these procedures and the FTIR was integrated into the DSH MOT timeleine.

An application was devised whereby 20 gases were monitored continuosly using a 5 minute averaging scheme: water vapor, carbon dioxide, carbon monoxide, nitrous oxide, methane, ethylene, pentane, toluene, o-xylene, pxylene, acetic acid, formaldehyde, acetaldehyde, acetone, methanol, ethanol, acetonitrile, acrylonitrile, dichloromethane, and ammonia. The FTIR was either physically relocated to a new location or the plumbing was changed so that a different location was monitored. The FTIR was zeroed using N2 gas every time it was relocated. The FTIR was operated from a PDA via Bluetooth connection. Data was downloaded to a PC on a daily basis.

\section{B. FTIR Objectives During DSH MOT}

The objectives of deploying the FTIR during the DSH MOT were:

1. Evaluate the air quality in several locations with the DSH.

2. To relocate the FTIR and to download data manually, and

3. To measure dynamic concentrations of selected gases at each location within DSH

4. To track patterns in $\mathrm{CO} 2$, ethanol, ammonia, pentane, and ethylene emissions back to exercise, sleep, and meal preparation events during the timeline of the mission.

\section{Results}

The current study presents results from the Veggie Plant Atrium and from the Hygiene Module. The average VOC concentrations observed at the Veggie Plant Atrium and in the Hygiene Module during the DSH MOT are shown in Fig 1. CH4 $(\sim 1.6 \mathrm{ppm})$ and $\mathrm{N}_{2} \mathrm{O}(\sim 0.35 \mathrm{ppm})$ are present in ambient air and they are essentially supplied by the air handler of the DSH. The highest average concentrations are ethanol, pentane, and ethylene (Hygiene), and carbon monoxide (Veggie). 


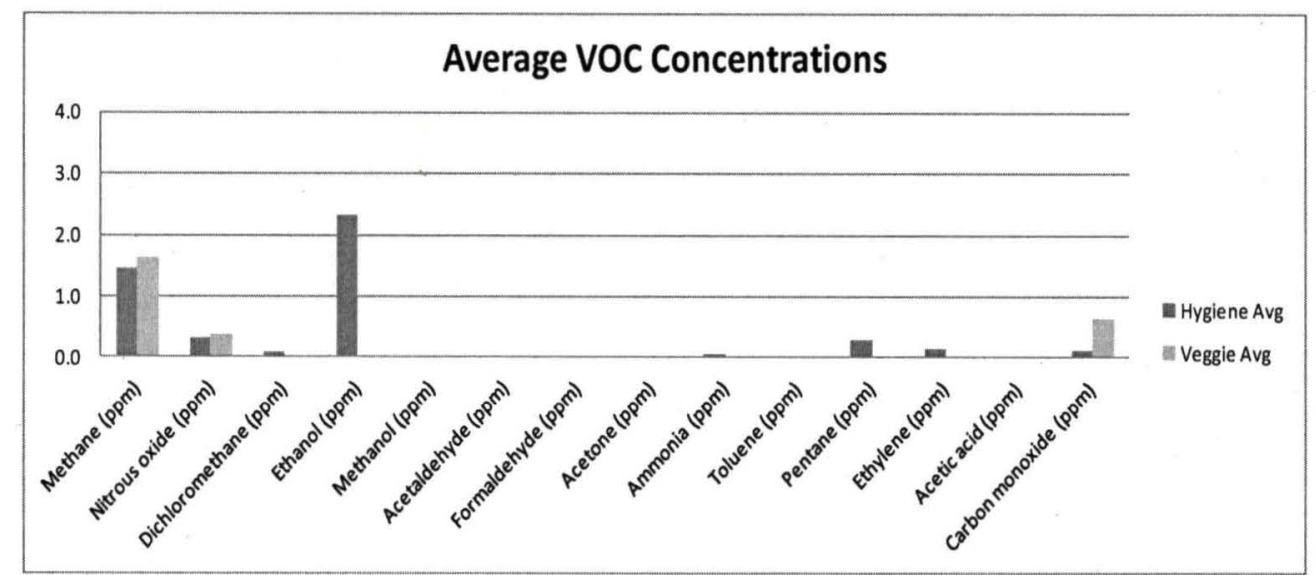

Fig 1. Average VOC concentrations observed in the Hygiene and Veggie locations during the DSH MOT.

The maximum VOC concentrations observed in the Veggie Plant Atrium and in the Hygiene Module during the DSH MOT are shown in Fig 2. The maximum concentration is more sensitive to detecting short duration changes in VOC concentration. Thus, we observe that ethanol concentration in the Hygiene module can be as high as $55 \mathrm{ppm}$. Other trace contaminants found in the Hygiene module include pentane $(20 \mathrm{ppm})$, dichloromethane $(8 \mathrm{ppm})$, acetaldehyde $(4 \mathrm{ppm})$, ammonia $(6 \mathrm{ppm})$, and ethylene $(7 \mathrm{ppm})$. Much lower concentrations of these same gases can be found in the Veggie Plant Atrium.
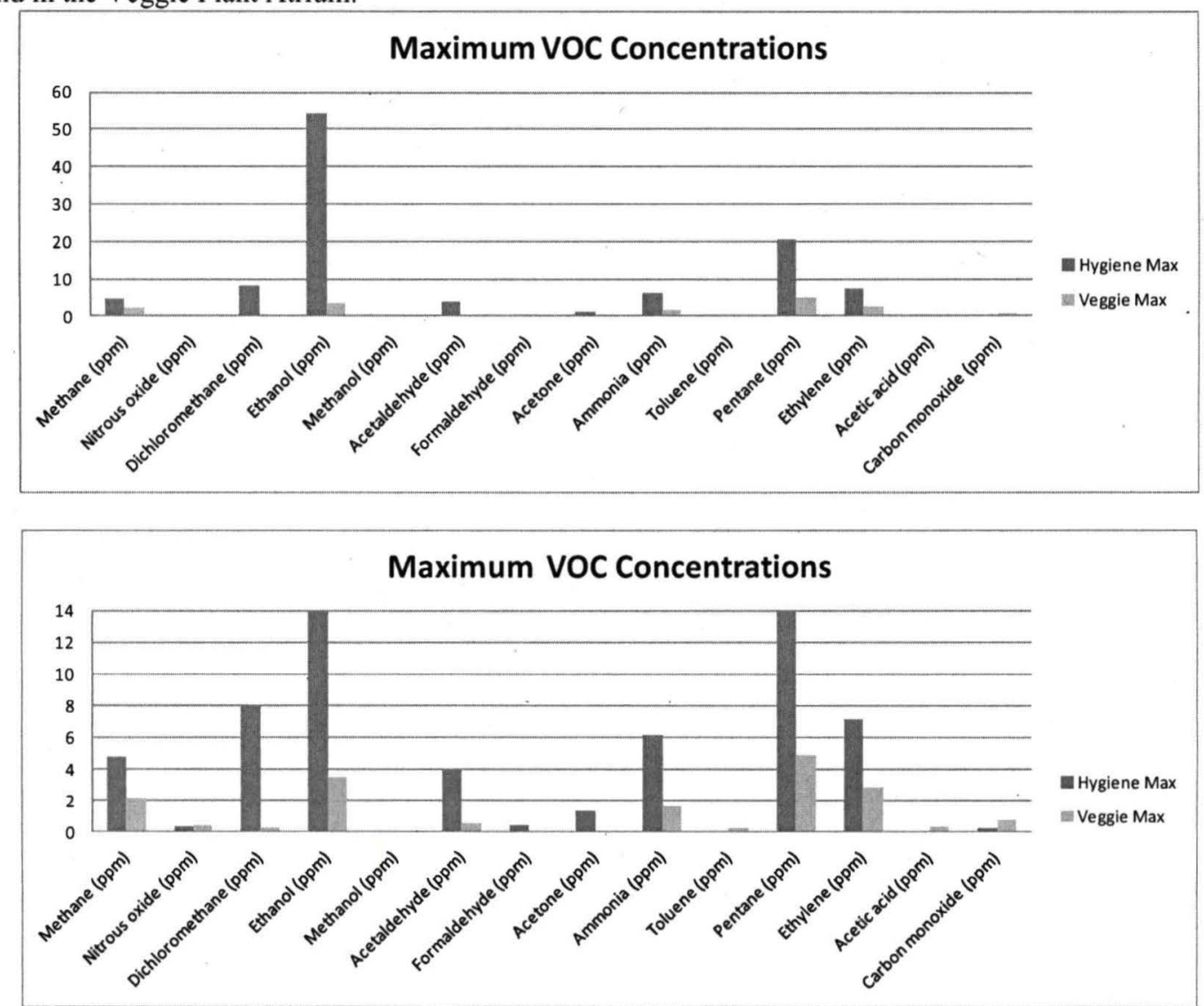

Fig 2. Maximum VOC concentrations observed in Hygiene and Veggie locations during the DSH MOT. 


\section{MOT Timeline - Veggie Plant Atrium}

During the MOT, the Veggie plant atrium was monitored for 3 days, however, the DSH was inhabited only during the $1^{\text {st }}$ day. The $1^{\text {st }}$ day the crew was asleep, they woke up at $6 \mathrm{AM}$, proceeded to exercise and then a meal was planned at 12 noon. The crew finished their activities at $4 \mathrm{pm}$ and the DSH was uninhabited for 2 days.

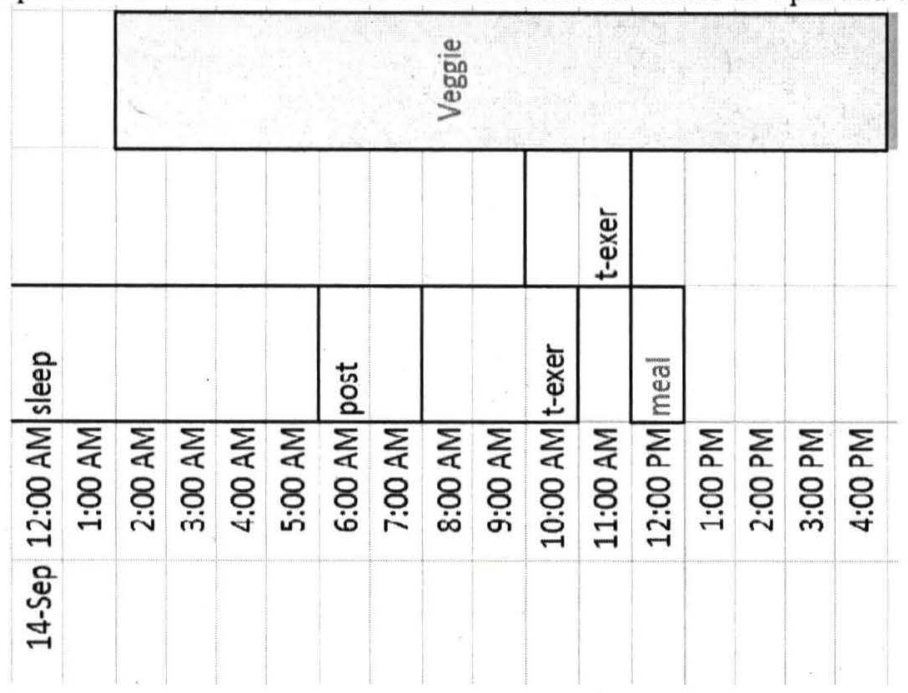

\section{Dynamic changes in trace contaminant concentrations during sampling of the Veggie Plant Atrium.}

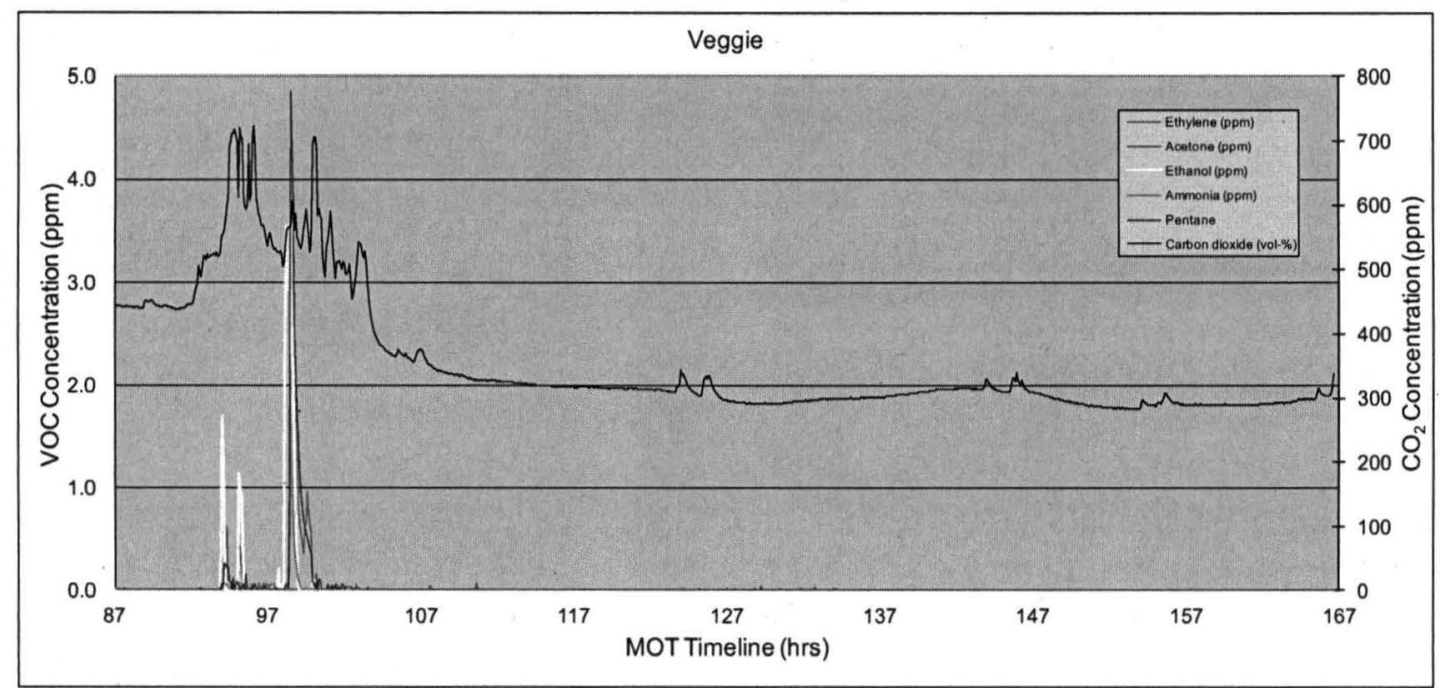

Fig 3. The $1^{\text {st }}$ day of the Veggie location illustrates dynamic changes associated with exercise and meal preparation during the DSH MOT (high concentrations of $\mathrm{CO} 2$, ethanol, pentane and ammonia). The next 2 days are void of human activities and the $\mathrm{CO} 2$ concentration drops to ambient levels.

Dynamic changes in VOC concentration show that the FTIR can track crew activities (exercise and cooking) taking place during the timeline (Fig 3). There were large increases in $\mathrm{CO}_{2}$ and ethanol concentrations when members of the crew exercised ( $\sim 90 \mathrm{hrs})$. A lunch meal took place at $\sim 100 \mathrm{hrs}$, which showed that pentane, ethylene, and ammonia were released during meal preparation (Fig 4). 


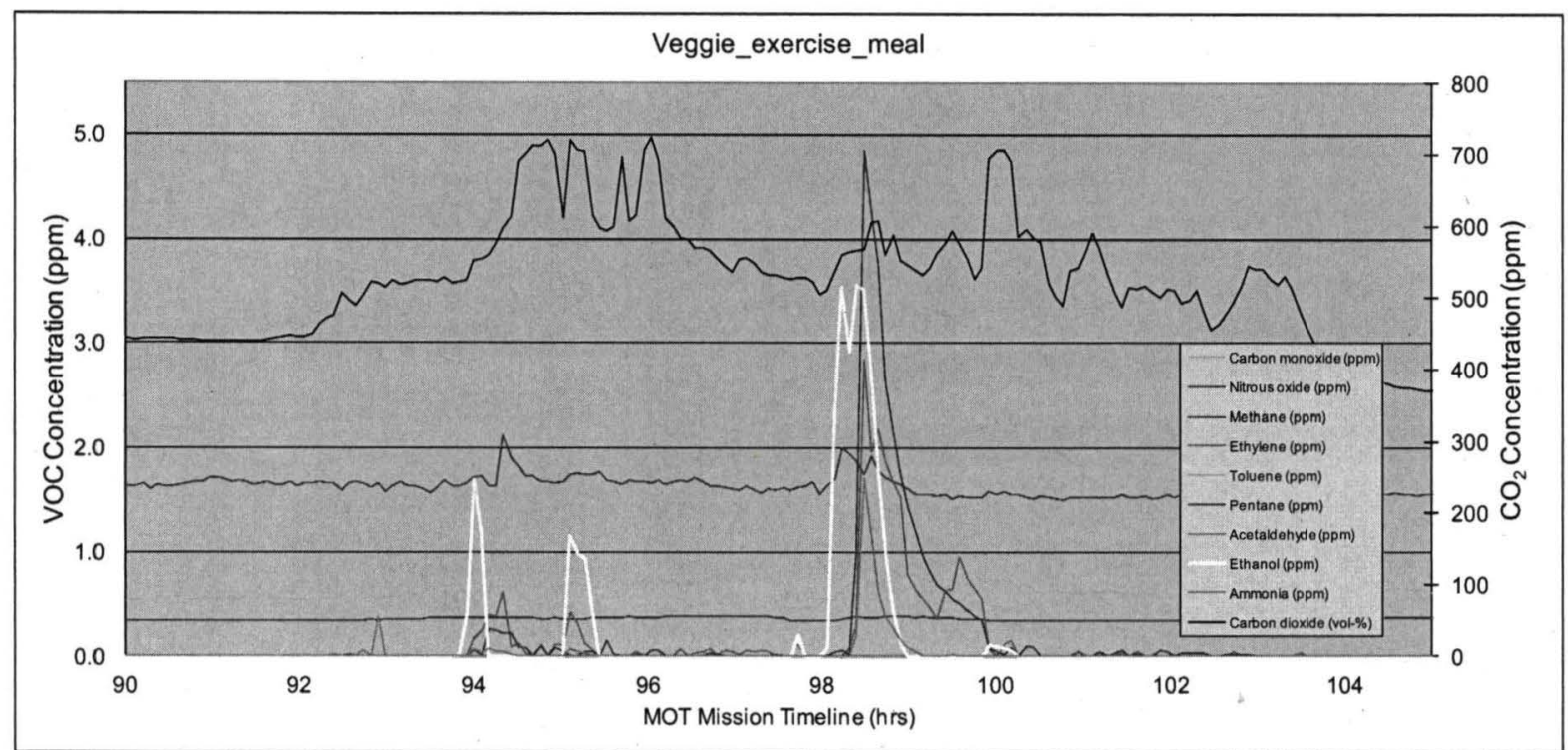

Fig 4. The $1^{\text {st }}$ day of the Veggie location illustrates dynamic changes associated with exercise and meal preparation during the DSH MOT (high concentrations of $\mathrm{CO} 2$, ethanol, pentane and ammonia).

The source of pentane during preparation of lunch is unknown, but pentane has been identified as a gas commonly emitted from polystyrene foam. Emissions of ethylene and ammonia were also observed at the same time. The ethanol and $\mathrm{CO} 2$ emissions are indicators of the exercise activities taking place in the DSH. The VOCs indicate that smaller emissions of ethylene and pentane are observed $\sim 94 \mathrm{hrs,} \mathrm{probably} \mathrm{associated} \mathrm{with} \mathrm{smaller} \mathrm{cooking} \mathrm{events}$ during breakfast.

\section{MOT Timeline - Hygiene Module}

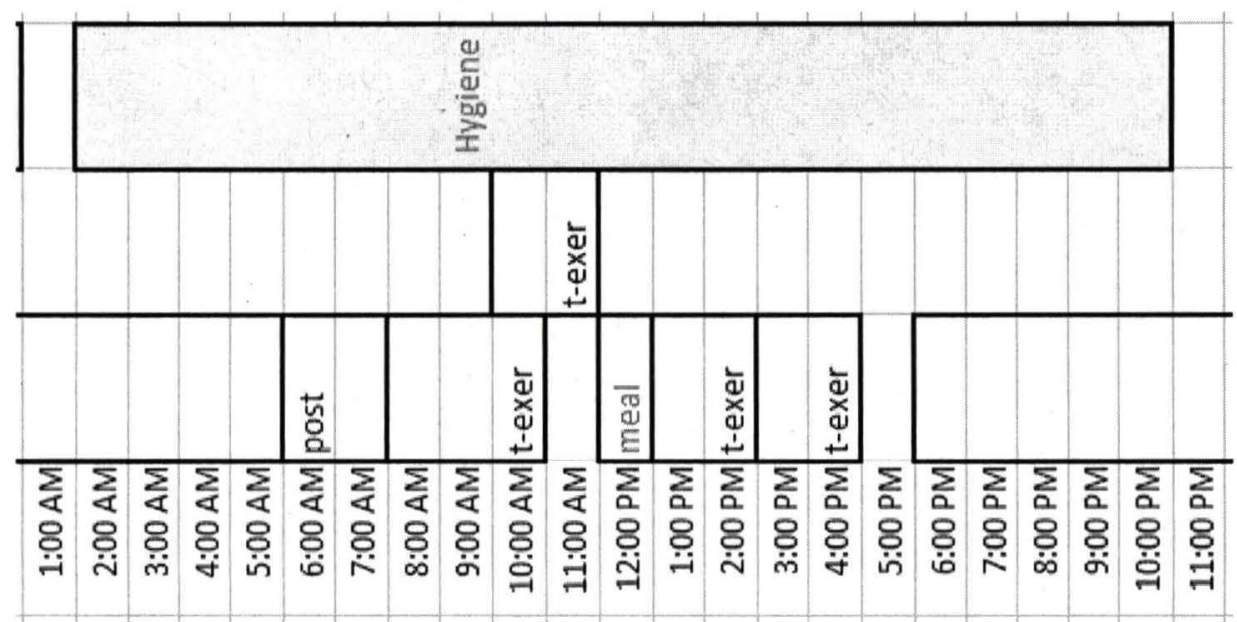

The FTIR was located in the Hygiene Module for an entire day. The Hygiene module is smaller and it is the bathroom of the DSH. The timeline of events coincided with exercise periods before and after a meal preparation event. Dynamic changes in VOC concentration in the Hygiene Module show that ethanol is used by the crew for hygiene and that large spikes ( $>30 \mathrm{ppm}$ ) of ethanol are observed (Fig 5). 
3. Dynamic changes in trace contaminant concentrations during sampling of the Hygiene Module.

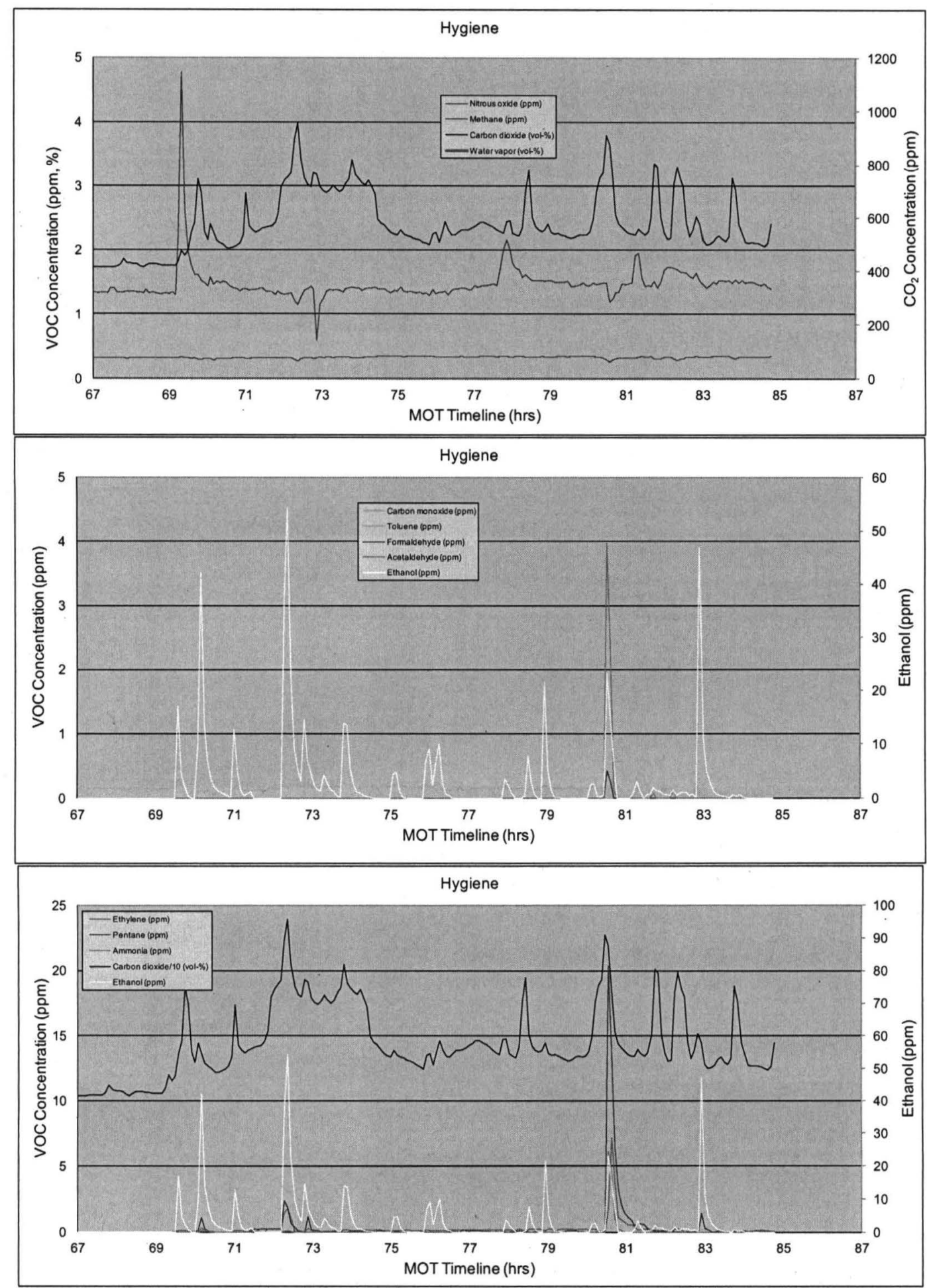

Fig 5. The VOC emissions (high concentrations of $\mathrm{CO}_{2}$, ethanol, pentane and ammonia) in the Hygiene Module illustrate dynamic changes associated with morning activities, exercise and meal preparation during the DSH MOT 


\section{Lessons learned}

- The procedures developed for operating the FTIR were successful as all data was collected and the FTIR worked during the entire MOT mission.

- The personnel were able to cope with small deviations from the training they received in part due to the procedures and training provided.

- Crew activities such as exercise, meal preparation, and hygiene options could de followed by watching peaks in certain VOCs: ethanol, $\mathrm{CO} 2$, pentane, $\mathrm{NH} 3$, and ethylene.

\section{Benefits to Habitation Concepts}

The dynamic measurements of habitat trace contaminants afforded by the portable FTIR show that crew activities like meal preparation, hygiene, and exercise result in short duration emissions of target VOCs. Hygiene and exercise events are characterized by spikes in $\mathrm{CO} 2$ and ethanol concentration. Meal preparation events, on the other hand, were characterized by short emissions of pentane, ammonia, and ethylene. The data shows that deploying an FTIR in each habitation module is warranted fior capturing all dynamic changes in VOCs in a habitat.

\section{Conclusion}

- The FTIR is ready for use in future DSH tests - successful operation, data collection and quantification of $>95 \%$ of gases in the FTIR application.

- Dynamic changes in concentration were detected during the MOT test.

- Having multiple FTIRs (one in each module) is preferred.

\section{Acknowledgments}

O. Monje would like to thank Gasmet for donating the use of the DX-4040 portable FTIR for use during vthe DSH MOT Test. The authors would also like to thank Tracy Gill at KSC and the MSFC ARREM AES Project for important contributions to the success of this study.

\section{References}

${ }^{1}$ Stuffler T, Mosebach H, Kampf D, Honne A, Schumann-Olsen H, Kaspersen K, Supper W, Tan G. ANITA Air Monitoring on the International Space Station Part 1: The Mission. SAE Technical Paper. 2008 Jun 29; 2008-01-2042. DOI: 10.4271/200801-2042.

${ }^{2}$ Honne A, Schumann-Olsen H, Kaspersen K, Clausen S, Mosebach H, Kampf D, Stuffler T, Supper W, Tan G. ANITA Air Monitoring on the International Space Station Part 2: Air Analyses. SAE International Journal of Aerospace. 2009; 1(1): 178192. DOI: $10.4271 / 2008-01-2043$.

${ }^{3}$ Honne A, Schumann-Olsen H, Kaspersen K, Limero TF., Macatangay AV, Mosebach H, Kampf D, Mudgett PD, James JT., Tan G, Supper W. Evaluation of ANITA Air Monitoring on the International Space Station. SAE International Journal of Aerospace. $2011 ; 4(1): 451-466$. DOI: 10.4271/2009-01-2520. 